\title{
Pretransition Phenomena in Fast-Proton Conductors
}

\author{
S. Waplak*, W. Bednarski and A. Ostrowski \\ Institute of Molecular Physics, Polish Academy of Sciences \\ Smoluchowskiego 17, 60-179 Poznań, Poland
}

\begin{abstract}
In this paper we present recent experimental results related to nonlinear crystal field fluctuations and current flow in $\mathrm{K}_{3} \mathrm{H}\left(\mathrm{SO}_{4}\right)_{2}$ fast-proton conductor far below the transition temperature $T_{\mathrm{g}}$. The results for crystalline and powdered samples are quantitatively explained in the framework of soliton model of charge transport.
\end{abstract}

PACS numbers: 76.30.--v, 66.30.-h

\section{Introduction}

The proton transfer along hydrogen-bonded channels has been recognized to be responsible for the charge conduction in many organic and biological systems [1-3]. Proton conducting solids are also of interest because of their potential use in fuel cells $[4,5]$. Current interest to fast-proton conductors needs their synthesis, parameterization, and especially understanding of charge transport mechanism on molecular level.

Since the basic work of Antonchenko, Davydov and Zolotaryuk (ADZ) [6] it has been widely believed that protons in hydrogen-bonds move in deformed double-well potential resulting from proton-lattice interactions. Combined with proton-proton interaction this characteristic provides the essential ingredients for the ionic defects to move as topological solitons.

The main aim of our work is to check experimentally a validity of data description in the framework of ADZ model. To get reproducible data we have collected a number of EPR and dc current flow data for $\mathrm{K}_{3} \mathrm{H}\left(\mathrm{SO}_{4}\right)_{2}$ (KHS) fastproton conductor pure and with $\mathrm{Mn}^{2+}, \mathrm{CrO}_{4}^{3-}$ paramagnetic ions in the temperature region below the transition to paraelectric fast-proton conducting phase $\left(T_{\mathrm{g}}=\right.$ $471 \mathrm{~K})$. We were motivated by Dolinšek et al. [7] NMR work where for $\mathrm{Rb}_{3} \mathrm{H}\left(\mathrm{SO}_{4}\right)_{2}$ so-called "pretransition phenomena" were observed about $100 \mathrm{~K}$ below $T_{\mathrm{g}}$. In such a way we avoided the sample decomposition and could repeat the experiments for

${ }^{*}$ corresponding author; e-mail: Stefan.Waplak@ifmpan.poznan.pl 
several times. The KHS crystal has a specific structure which contains a "zero-dimensional" hydrogen-bond network consisting of isolated $\mathrm{SO}_{4}^{2-}-\mathrm{H} \ldots \mathrm{SO}_{4}^{2-}$ dimers, which can be treated as non-interacting.

By means of EPR on paramagnetic ions placed in different sites of crystal lattice we can look closely on their response on charge transport.

This paper is organized as follows: Sect. 2 contains a short KHS sample description and preparation, Sect. 3 - short description of an experimental procedure, Sect. 4 - EPR data for KHS: $\mathrm{Mn}^{2+}$ and $\mathrm{KHS}$ : $\mathrm{CrO}_{4}^{3-}$, Sect. $5-$ dc current versus time data, Sect. 6 - soliton model overview, Sect. 7 - discussion and conclusions.

\section{Sample description and preparation}

X-ray data $[8]$ show that KHS at room temperature belongs to a space group $A 2 / a, Z=4$ with $a=9.777 \AA, b=5.674 \AA, c=14.667 \AA$ and $\beta=102.97$ lattice parameters. Below $471 \mathrm{~K}$, KHS exhibits ferroelastic properties and above $471 \mathrm{~K}$ it reveals a high protonic conductivity order of $10^{-2} \Omega^{-1} \mathrm{~cm}^{-1}$ [9]. Hexagonal plate-like crystals under consideration were grown isothermally at $295 \mathrm{~K}$ from saturated aqueous solution containing 19 wt. $\% \mathrm{~K}_{2} \mathrm{SO}_{4}, 12.7$ wt. $\% \mathrm{H}_{2} \mathrm{SO}_{4}$, and an order of 0.3 wt. $\%$ of the salt of paramagnetic ions: $\mathrm{Mn}^{2+}$ or $\mathrm{CrO}_{4}^{2-}$. Optical inspection of the single crystals (pure or doped) in polarized light reveals the ferroelastic domain pattern vanished at $471 \mathrm{~K}$. At about $518 \mathrm{~K}$ samples were melt. The spin concentration in doped samples was of the order of 500-1000 ppm.

The samples used to study of the influence of an external electric fields on EPR or current flow parameters were prepared in the form of plates $0.7-1.1 \mathrm{~mm}$ thin perpendicular to the $c$-axis of KHS with crystal volume of about $12 \mathrm{~mm}^{3}$ with silver electrodes applied.

\section{Experimental procedure}

The electric circuit used to study of current flow under dc an external electric field is shown in Fig. 1. The EPR resonator contained the capacitor with the sample as well as a system of temperature control and stabilization was able to

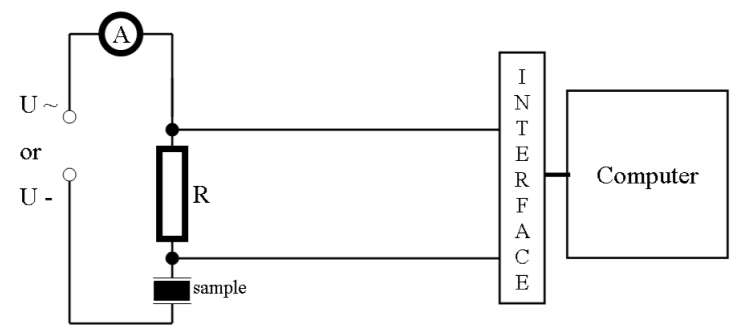

Fig. 1. The electric circuit used to study of current flow. 
operate in the temperature range of 80-500 K. This system allowed us to record the EPR spectra and/or dc current data.

\section{EPR data for KHS: $\mathrm{CrO}_{4}^{3-}$ and $\mathrm{KHS}: \mathrm{Mn}^{2+}$}

The samples with $\mathrm{CrO}_{4}^{3-}(S=1 / 2)$ paramagnetic centers where grown from a mother solution used to pure KHS crystal growth and an additionally 0.3 wt.\% of $\mathrm{K}_{2} \mathrm{CrO}_{4}$ was added. Detailed EPR data were published elsewhere [10]. The essential results are:

1. the existence of translational diffusion of protons between well-defined lattice sites;

2. rotation of $\mathrm{SO}_{4}^{2-}$ units very well reproduced by $\mathrm{CrO}_{4}^{3-}$ units which replace $\mathrm{SO}_{4}^{-2}$. As a result of tetrahedra rotation, EPR records a superhyperfine structure from two nonequivalent protons and in high temperature $\mathrm{CrO}_{4}^{3-}$ transforms to $\mathrm{H}_{2} \mathrm{CrO}_{4}^{-1}$.

$\mathrm{Mn}^{2+}$ ion $(S=5 / 2)$ replaces a potassium ion in KHS in octahedral coordination of six oxygens from neighboring $\mathrm{SO}_{4}$ groups. The excess charge $(+1)$ of $\mathrm{Mn}^{2+}$ is compensated by the proton vacancy. There is a reason why $\mathrm{Mn}^{2+}$ in KHS is an excellent probe for monitoring of proton motion which directly modulates the crystal field EPR parameters. In Fig. 2 the $( \pm 3 / 2 \leftrightarrow \pm 1 / 2)$ linewidth tem-

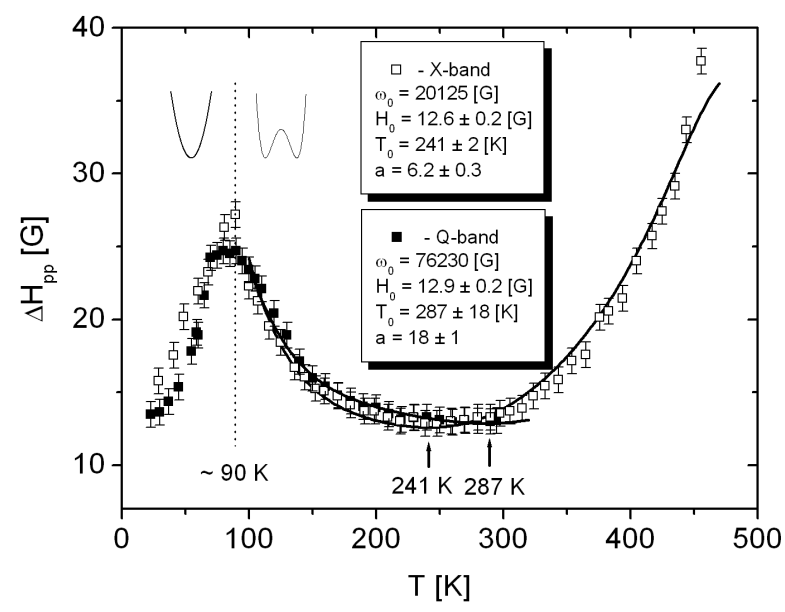

Fig. 2. Temperature dependence of $\Delta H_{\mathrm{pp}}$ linewidth of $( \pm 3 / 2 \leftrightarrow \pm 1 / 2)$ transition in KHS: $\mathrm{Mn}^{2+}$ at X- and Q-band.

perature dependence in the region of $20-470 \mathrm{~K}$ is presented. The first linewidth anomaly at about $90 \mathrm{~K}$ reflects a transition from single to double minimum of proton potential [11]. Above this temperature the linewidth versus temperature can be described by fluctuation of crystal field on $\mathrm{Mn}^{2+}$ complex evoked by "inter" and "intra-bond" proton motion in the following form: 


$$
\Delta H_{\mathrm{pp}}(T)=\Delta H_{\mathrm{pp}}^{0}+(2 M-1) \frac{D^{2}(T)}{\omega_{0}}\left(\frac{T-T_{0}}{T_{0}}\right)^{2},
$$

where $D$ is the fine structure crystal-field parameter, $\omega_{0}$ is the EPR frequency.

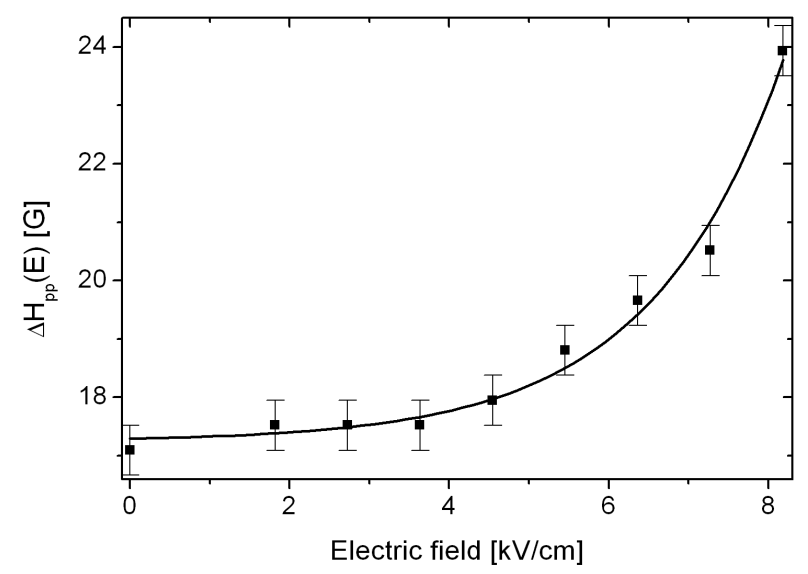

Fig. 3. The linewidth $\Delta H_{\mathrm{pp}}( \pm 3 / 2 \leftrightarrow \pm 1 / 2)$ vs. an external electric field in KHS:Mn ${ }^{2+}$ at $360 \mathrm{~K}$.

Because the linewidth at about $460 \mathrm{~K}$ is still sufficiently narrow (of about $30 \mathrm{G}$ ) these data once again exclude random proton liquid-like diffusion even near below $T_{\mathrm{g}}$ temperature. Usually in dielectric materials without a long range order values of an external field applied to influence EPR spectra are of an order of hundred $\mathrm{kV}$. It is not the case for KHS: $\mathrm{Mn}^{2+}$ above about $360 \mathrm{~K}$ as shown in Fig. 3. In accordance with Chen et al. [12] the KHS conductivity at about $360 \mathrm{~K}$ is of an order of $10^{-5} \Omega^{-1} \mathrm{~cm}^{-1}$. An external dc field above about $4 \mathrm{kV} / \mathrm{cm}$ leads to nonlinearity in the linewidth behavior strictly connected with the current flow.

\section{DC current versus time}

Figure 4 presents the time evolution of voltage/current measured in circuit (Fig. 2) on resistance $R_{1}=10 \mathrm{k} \Omega$ at $440 \mathrm{~K}$ under dc field equal $51 \mathrm{~V}$. The current peaks direction up/down depend on the electrodes polarity.

We have obtained such a typical characteristic for pure as well as for lightly doped KHS with $\mathrm{Mn}^{2+}$ or $\mathrm{CrO}_{4}^{-3}$ ions. The current value for the peak of maximal intensity $I_{\max }$ is about $250 \mu \mathrm{A}$ in comparison with the base current $I_{0}=30 \mu \mathrm{A}$ for sample $1.3 \mathrm{~mm}$ thick plates and grows up with the sample thickness.

Next similar experiments have been done for powdered sample and the results are shown in Fig. 5. Here $I_{\max } \approx 3 \mu \mathrm{A}$ inside the spectrum of noise.

Figure 6 displays the current time evolutions for two parts of the same KHS sample: $a$ - for the fresh sample at $403 \mathrm{~K}, b$ - for the sample heated above 


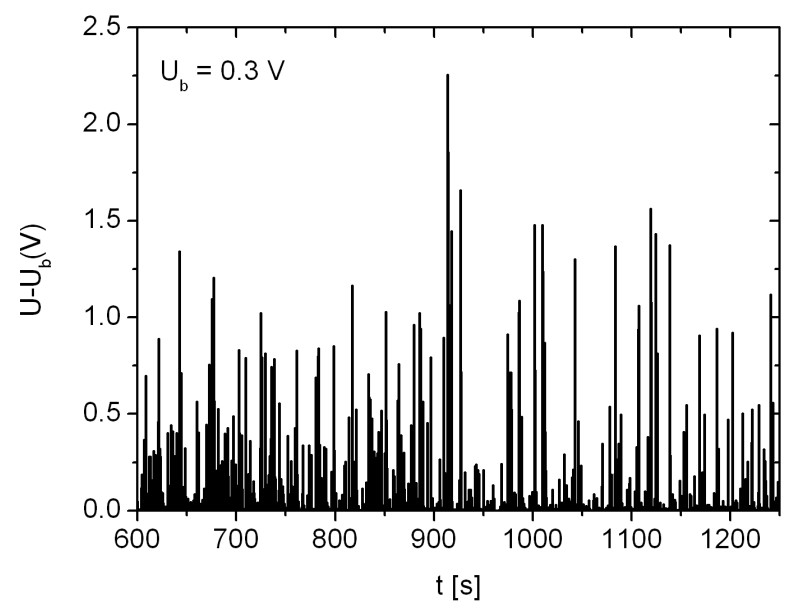

Fig. 4. The current/voltage changes vs. time for $\mathrm{KHS}_{\mathrm{Cr}}{ }^{6+}$ at $440 \mathrm{~K}$ under $51 \mathrm{~V}$ dc field $U_{\mathrm{b}}$ is the basic voltage at the moment when current peaks are not present.

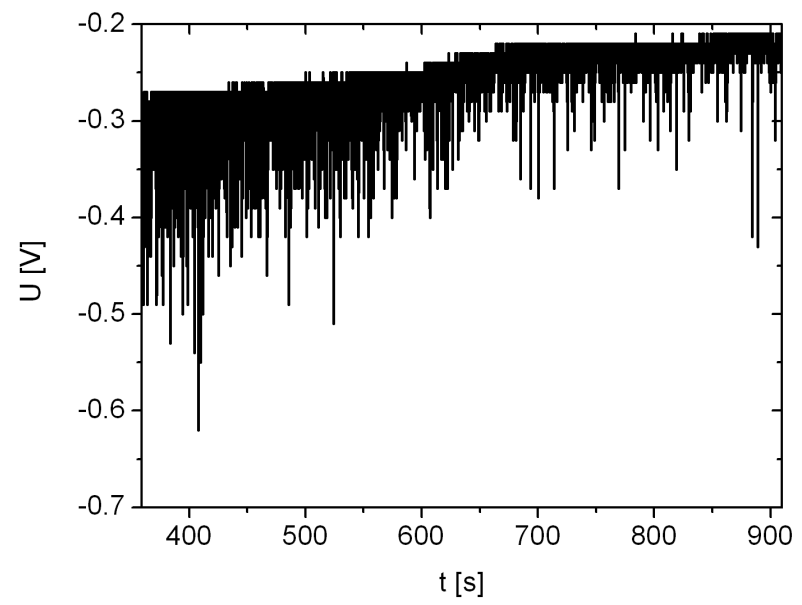

Fig. 5. The current/voltage changes vs. time for powdered KHS at $430 \mathrm{~K}$ under $53 \mathrm{~V}$ dc field.

$T_{\mathrm{g}}=471 \mathrm{~K}$ and quickly cooled down to $403 \mathrm{~K}$. Notice that the sample heated above $T_{\mathrm{g}}$ do not reveal noticeable current peaks.

In the next experiment a sample was heated above $T_{\mathrm{g}}$ up to $475 \mathrm{~K}$ and $12 \mathrm{~V}$ was applied for a few minutes. Next the sample was quickly cooled down to $460 \mathrm{~K}$ and dc field was applied by step of $10 \mathrm{~V}$.

For dc field $U \geq 10 \mathrm{~V}$ current peaks appear again and their heights were proportional to dc field value in nonlinear manner. Figure 7 shows the height of current peak versus dc field. The data presented in Figs. 5-7 allow us to conclude that:

1. current fluctuation is not evoked by the grain in powdered samples, 


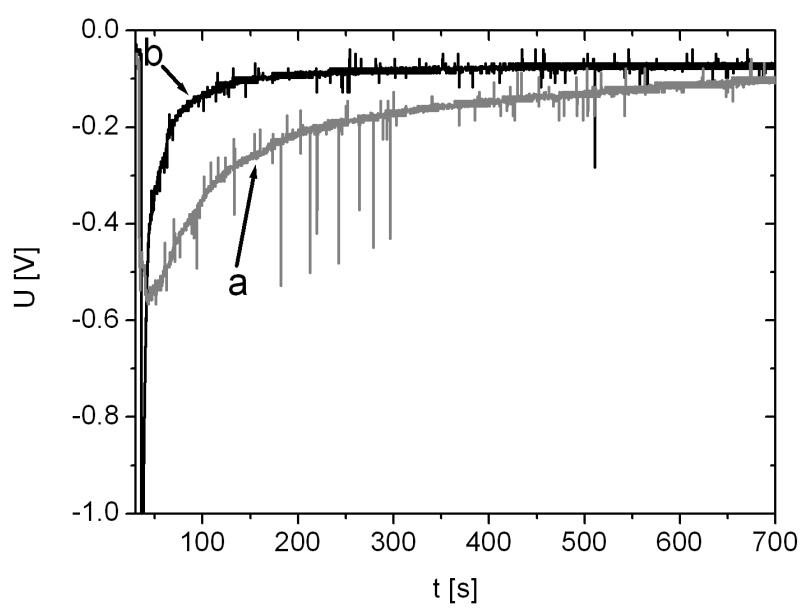

Fig. 6. The voltage/current changes vs. time for two parts of KHS: $\mathrm{Mn}^{2+}$ samples, $a$ - before heating above $T_{\mathrm{g}}=471 \mathrm{~K}, b$ - after heating above $T_{\mathrm{g}}$. Both data were collected at $403 \mathrm{~K}$ under $49.5 \mathrm{~V}$ dc field.

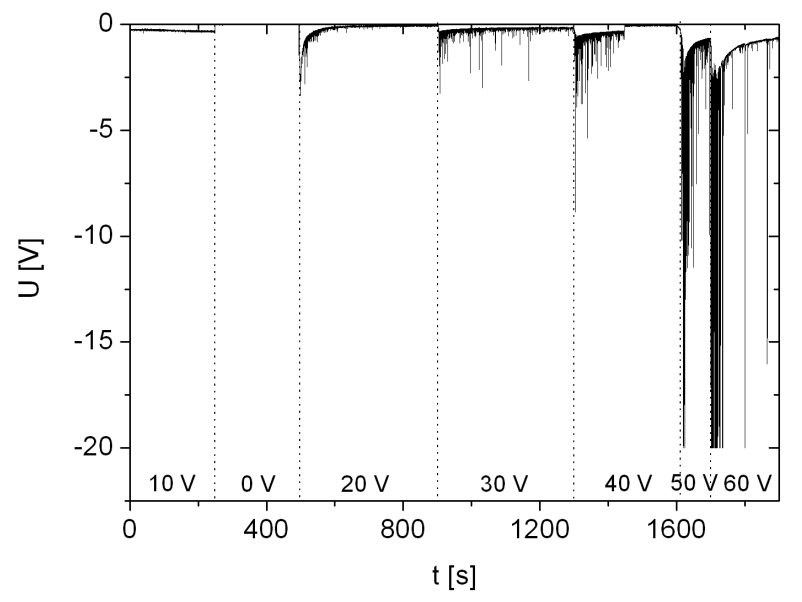

Fig. 7. The current/voltage dependence vs. time for the sample heated up to $475 \mathrm{~K}$ with dc $12 \mathrm{~V}$, cooled down to $460 \mathrm{~K}$ (without field) and next measured under dc field by step of $10 \mathrm{~V}$.

2. for crystalline samples a current polarization above $T_{\mathrm{g}}$ preserves current flow below $T_{\mathrm{g}}$ with some critical external electric field value.

Kamimura et al. [13] calculate the conductivity of KHS below the phase transition temperature. In accordance with their data the ionic current is expressed in the following form:

$$
I=q \lambda_{\mathrm{s}} \alpha U
$$

where $q$ is the electric charge, $U$ is the electric field, $\alpha$ is the proton mobility, $\lambda_{\mathrm{S}}$ is 
the wide of "stripe domains" the or the length of zig-zag proton path and below $T_{\mathrm{g}}$

$$
I \sim\left(T_{\mathrm{g}}-T\right)^{-\frac{1}{2}}
$$

It is considered that the current value versus KHS takes a new phase in the presence of an applied electric field. In this phase there exist the "stripe domains" in which the distances between $\mathrm{SO}_{4}$ octahedra are the same and the intervening region in which $\mathrm{SO}_{4}$ groups still form the hydrogen-bonded dimers. These two regions appear alternately and form "stripes". It looks that only after stripes are founded irregular current flow may be observed. A theory of soliton scattering by "impurities" in hydrogen-bonded chains was derived by Kivshar [14] as an extending of ADZ model, which we shortly overview below.

\section{Soliton model overview}

The soliton model of proton transfer proposed by Antonchenko et al. [6] (ADZ model) has a following basic idea: The proton in each hydrogen bond of the chain can occupy one of two equilibrium positions separated by a potential barrier whose height strongly depends on distance between adjacent $(\mathrm{O}-\mathrm{O}$ in our case) atoms. A coupling motion of the protons and $\mathrm{O}$ atoms provide a barrier lowering mechanism that allows an easy proton transfer between two equilibrium protons positions. The coupling between a vibrational proton mode and an optical mode of the heavy ionic sublattice are included in this model.

The effect of protons and heavy ion coupling reduces the effective potential barrier to the following form:

$$
\varepsilon=\varepsilon_{0}\left(1-\frac{\chi^{2} u_{0}^{4}}{2 M \Omega_{0}^{2}}\right)
$$

where $\chi$ is the proton-heavy ion coupling, $2 u_{0}$ is the distance between two minima of double-wall potential $E_{0}, M$ is the ion mass, $\Omega_{0}$ is the frequency of optical mode.

The solution of coupled equations for proton motion ADZ model has been done for particular cases:

1. vanishing of coupling $(\chi \rightarrow 0)$,

2. for soliton velocity $v \approx v_{0}$, where $v_{0}=a \Omega_{1}$ is the characteristic velocity arising from dispersion mode in the $\mathrm{X}$ (oxygen) sublattice. Then the kink solutions are as follows:

$$
\begin{aligned}
& u=u_{0} \tanh \left(\frac{\xi}{L}\right) \quad \text { for proton sublattice, } \\
& \rho=\rho_{0} \operatorname{sech}^{2}\left(\frac{\xi}{L}\right) \quad \text { for oxygen sublattice, }
\end{aligned}
$$

where $\xi=(x-v t) / a, 1 / L^{2}=\left(2 \varepsilon / m \omega_{1}^{2} u_{0}^{2}\right) 1 /\left[1-\left(v / v_{0}\right)^{2}\right]$ and $x$ is the normal 
coordinate, $m$ is the proton mass, $\omega_{1}$ is the frequency of acoustic mode. The only numerical solutions are possible for $v \neq v_{0}$. In an external electric field solitary wave should be accelerated with some velocity $v$ and large-amplitude oscillations should be emitted in oxygen sublattice reducing $v \rightarrow v_{0}$. Our data strongly suggest that irregularity of current flow observed comes from soliton scattering on "striples". Kivshar [14] claims that when a soliton changes its velocity due to scattering, it generates radiation, i.e. emission in the hydrogen and oxygen subsystem. Figure 8 shows the evolution of high current peaks for 65000 probes

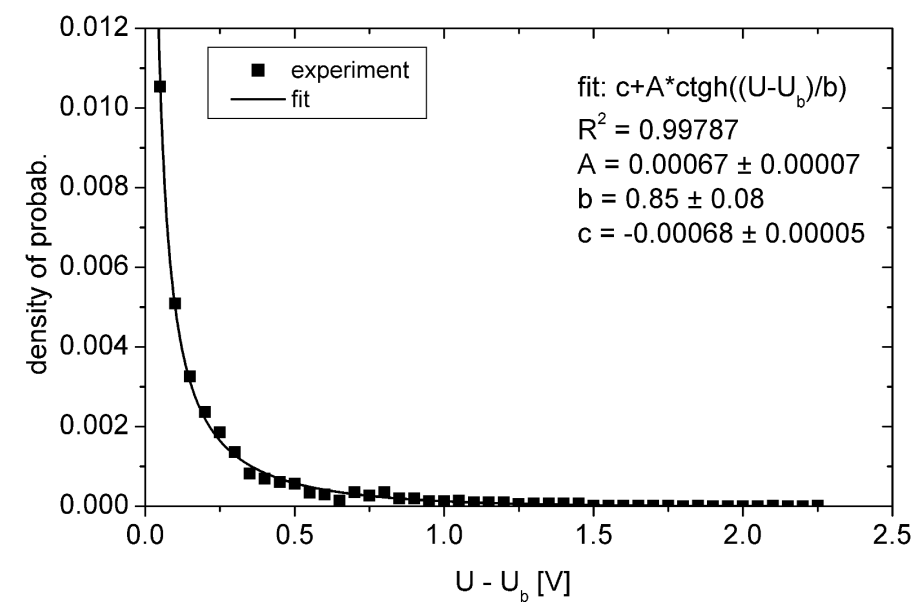

Fig. 8. Probability of height current/voltage peak distribution for 65000 probes collected during $650 \mathrm{~s}$ from the data shown in Fig. 4.

collected during $650 \mathrm{~s}$. The peak amplitudes during this period time are described by the following equation:

$$
p=c+A \operatorname{ctgh} \frac{\left(U-U_{\text {basic }}\right)}{b} .
$$

This equation may be obtained from Eq. (4a):

$$
p \propto \frac{\partial}{\partial t} u_{n}, \quad p=\operatorname{Actgh}\left(\frac{\xi}{L}\right)
$$

where $L \propto \Delta U$ and displays an energy emitted by current flow.

In the context of a simplified soliton model the protonic kinks are accompanied by compression on refractions of the heavy sublattice around the protonic defects (stripes) carrying a pulse proton-electron charge.

\section{Discussion and conclusions}

Protons under dc field move along the chain making ionic defects $\mathrm{SO}_{4}^{(-\mathrm{e})}$ and $\mathrm{H}_{2} \mathrm{SO}_{4}^{(+\mathrm{e})}$ (due to rotation) Bjerrum defects. After passing of ionic defect to 
the end of chain the transport process start to beginning due to Bjerrum defect. In result the condition $e_{I}+e_{B}=e$ is satisfied. In the context of this simplified model, we have the following physical interpretations for protonic kink soliton solution [15]:

Kink $\mathrm{I} \rightarrow I^{-}$ionic defect,

Kink II $\rightarrow L$ Bjerrum defect,

Antikink $\mathrm{I} \rightarrow I^{+}$ionic defect,

Antikink II $\rightarrow D$ Bjerrum defect.

Soliton velocity $v$ (accelerated by dc field) modulates the height of the potential barriers. However, when $v \sim v_{0}$ two sublattice couplings have the essential effects on the defect dynamics and will produce irregularities in the mobility properties of the soliton (charge) excitations [16].

After ADZ model was published a number of papers have appeared with the aim to perfect this idea, nevertheless the number of parameters used in is hard to get experimentally.

We have shown that our experimental data are in qualitative agreement with ADZ theory. Further experiments also under ac drive are in progress.

\section{Acknowledgment}

This work is partially supported by grant Nr. 1 P03B 08027 and by the Centre of Excellence for Magnetic and Molecular Materials for Future Electronics within the European Commission No. G5MA-CT-2002-04049.

\section{References}

[1] X. Wang, D.W. Brown, K. Lindenberg, B.J. West, Phys. Rev. A 37, 3557 (1998).

[2] J.F. Nagle, M. Millia, H.J. Morowitz, J. Chem. Phys. 2, 3959 (1980).

[3] H. Merz, G. Zundel, Biochem. Biophys. Res. Commun. 101, 540 (1981).

[4] S.M. Haile, D.A. Boysen, C.R.I. Chisholm, R.B. Merle, Nature 410, 910 (2001).

[5] Y. Matsuo, K. Saito, H. Kawashima, S. Ikehata, Solid State Commun. 130, 414 (2004).

[6] V.Ya. Antonchenko, A.S. Davydov, A.V. Zolotaryuk, Phys. Status Solidi B 115, 631 (1983).

[7] J. Dolinšek, V. Mikac, J.E. Javoršek, G. Lahajnar, R. Blinc, Phys. Rev. B 58, 8445 (1998).

[8] Y. Noda, S. Vehiyama, K. Kafuku, H. Kasatani, H. Terauchi, J. Phys. Soc. Jpn. 59, 2804 (1990). 
[9] Y. Matsuo, J. Hatori, Y. Nakashima, S. Ikehata, Solid State Commun. 130, 269 (2004).

[10] S. Waplak, W. Bednarski, A. Ostrowski, J. Phys. Chem. Sol. 64, 229 (2003).

[11] Y. Noda, H. Kasatani, Y. Watanabe, H. Terauchi, J. Phys. Soc. Jpn. 61, 905 (1992).

[12] R.H. Chen, R.Y. Chang, C.S. Shern, T. Fukami, J. Phys. Chem. Sol. 64, 553 (2003).

[13] H. Kamimura, S. Watanabe, Philos. Mag. B 81, 1012 (2001).

[14] Y.S. Kivshar, Phys. Rev. A 43, 3117 (1991).

[15] S. Pnevmatikos, Phys. Rev. Lett. 60, 1534 (1988).

[16] G.P. Tsironis, S. Pnevmatikos, Phys. Rev. B 39, 7161 (1989). 